\title{
Quantum chemical modeling of molecular crystal deformations
} Yevhenii A. Vaksler ${ }^{1,2,3, *}$, Abdenacer Idrissi ${ }^{2}$, and Svitlana V. Shishkina ${ }^{1,3}$

${ }^{1}$ V.N. Karazin Kharkiv National University, 4 Svobody Sq., Kharkiv, 61022, Ukraine ${ }^{2}$ SSI "Institute for Single Crystals" NAS of Ukraine, 60 Nauky ave., Kharkiv, 61001, Ukraine

${ }^{3}$ Laboratoire de Spectroscopie pour les Interactions, la Réactivité et L'environnement (UMR CNRS A8516), Université de Lille, 59655, Villeneuve d'Ascq Cedex, France. * Corresponding author: vakslerea@gmail.com
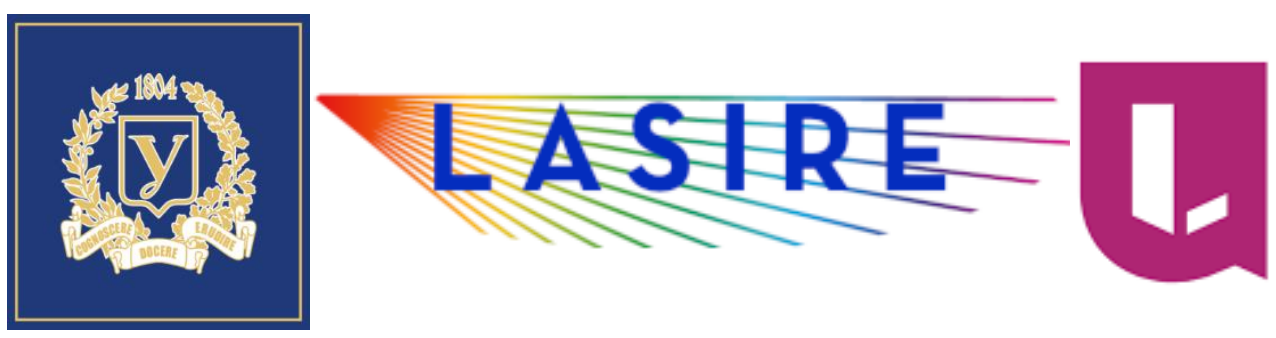

Université de Lille

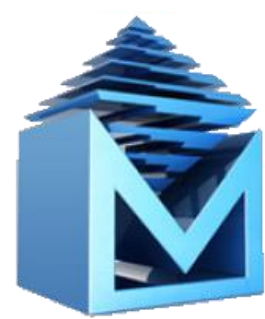


Energy-vector diagrams for visualization

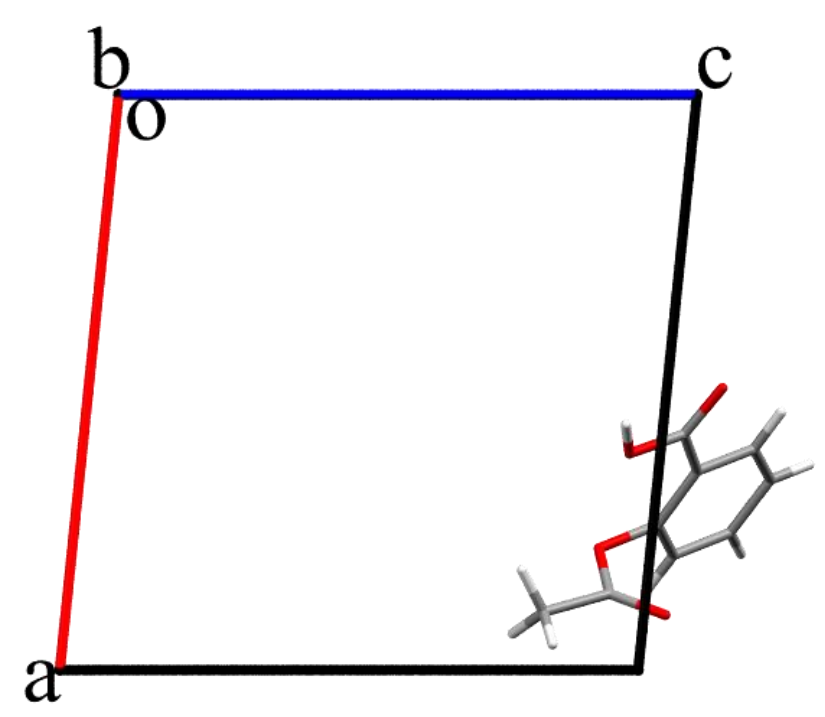

Crystals 


\section{Energy-vector diagrams for visualization}

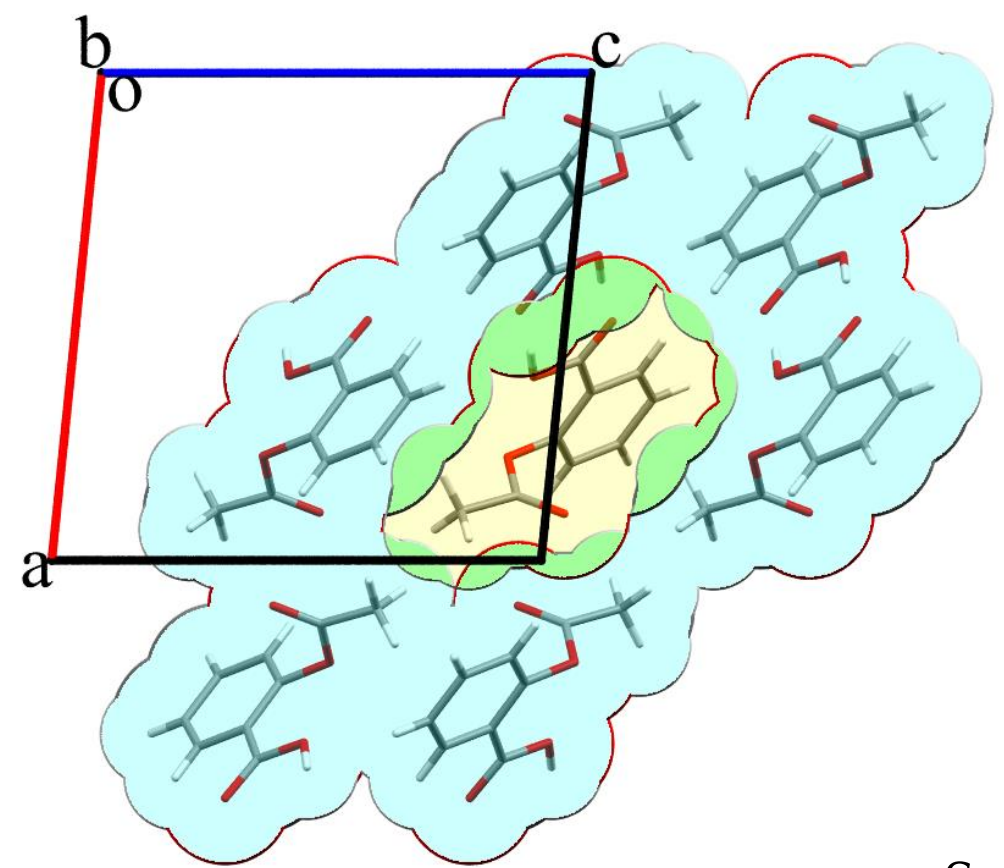

Search for neighbors:

$D_{i j} \leq V d W_{i}^{i m}+V d W_{j}^{n m}+1$

Crystals where $D_{i j}$ - distance between the $\mathrm{i}$ and $\mathrm{j}$ atoms of the initial molecule and $\mathrm{n}$ th neighbor $V d W_{i}^{i m}$ - van der Waals radius of the $i$ atom of the initial molecule $V d W_{j}^{n m}$ - van der Waals radius of the $\mathrm{j}$ atom of the neighboring molecule 


\section{Energy-vector diagrams for visualization}

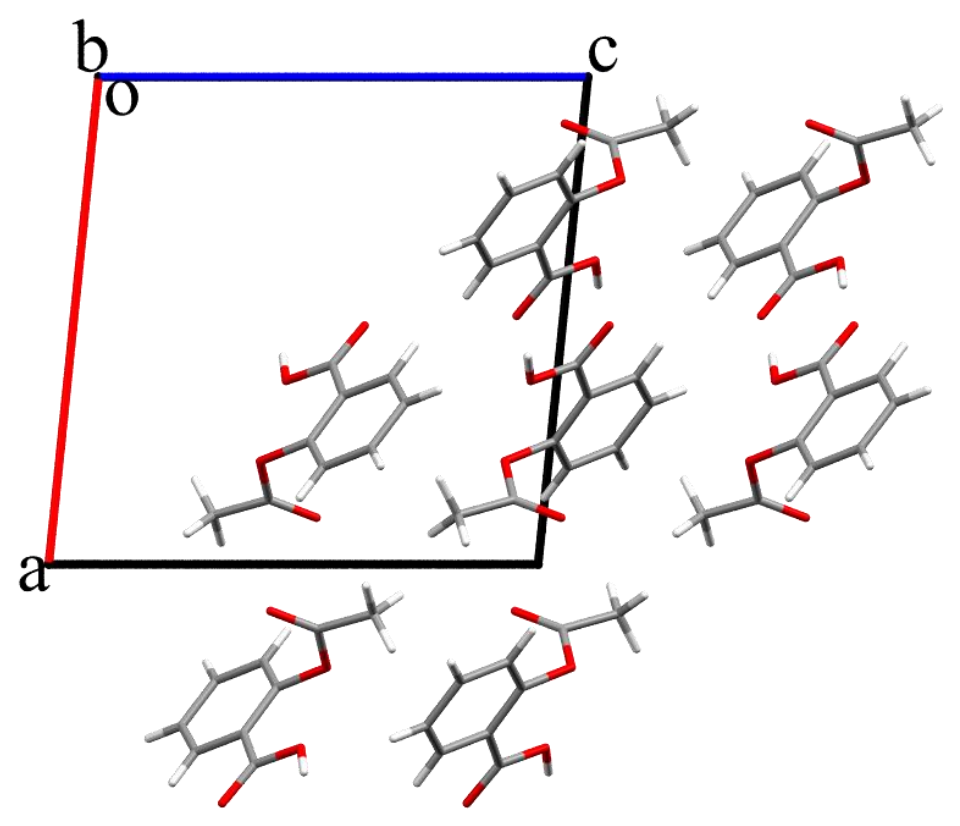

Search for neighbors:

$$
D_{i j} \leq V d W_{i}^{i m}+V d W_{j}^{n m}+1
$$
where $D_{i j}$ - distance between the $i$ and $j$ atoms of the initial molecule and $n$th neighbor $V d W_{i}^{i m}$ - van der Waals radius of the $i$ atom of the initial molecule $V d W_{j}^{n m}$ - van der Waals radius of the $\mathrm{j}$ atom of the neighboring molecule 


\section{Energy-vector diagrams for visualization}

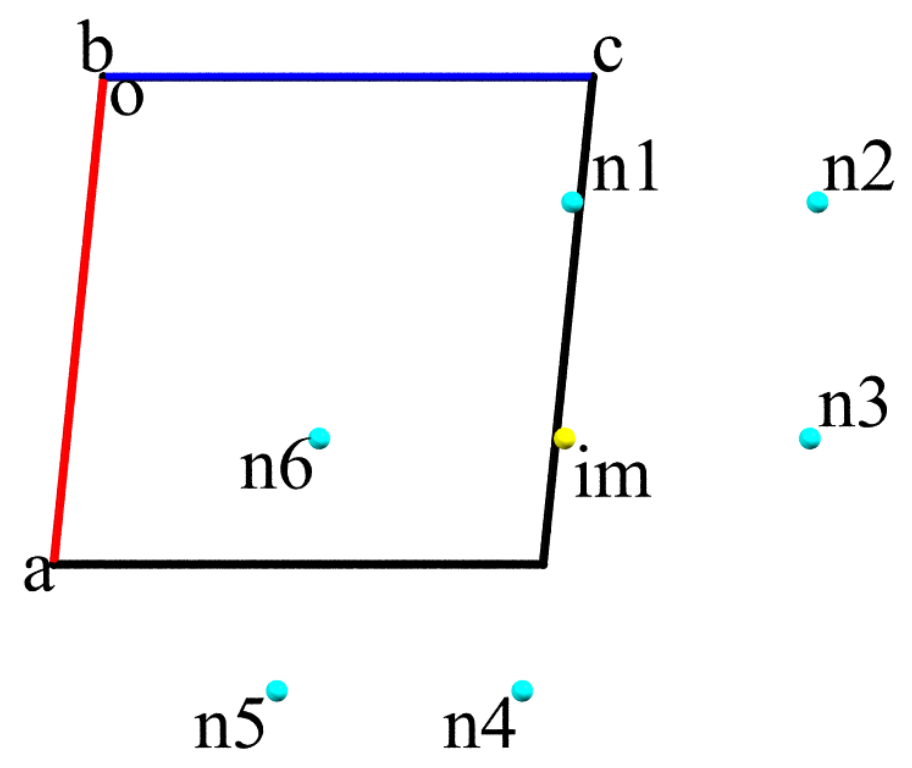

Search for neighbors:

$$
D_{i j} \leq V d W_{i}^{i m}+V d W_{j}^{n m}+1
$$
where $D_{i j}$ - distance between the $i$ and $j$ atoms of the initial molecule and $n$th neighbor $V d W_{i}^{i m}$ - van der Waals radius of the $i$ atom of the initial molecule $V d W_{j}^{n m}$ - van der Waals radius of the $\mathrm{j}$ atom of the neighboring molecule 


\section{Energy-vector diagrams for visualization}

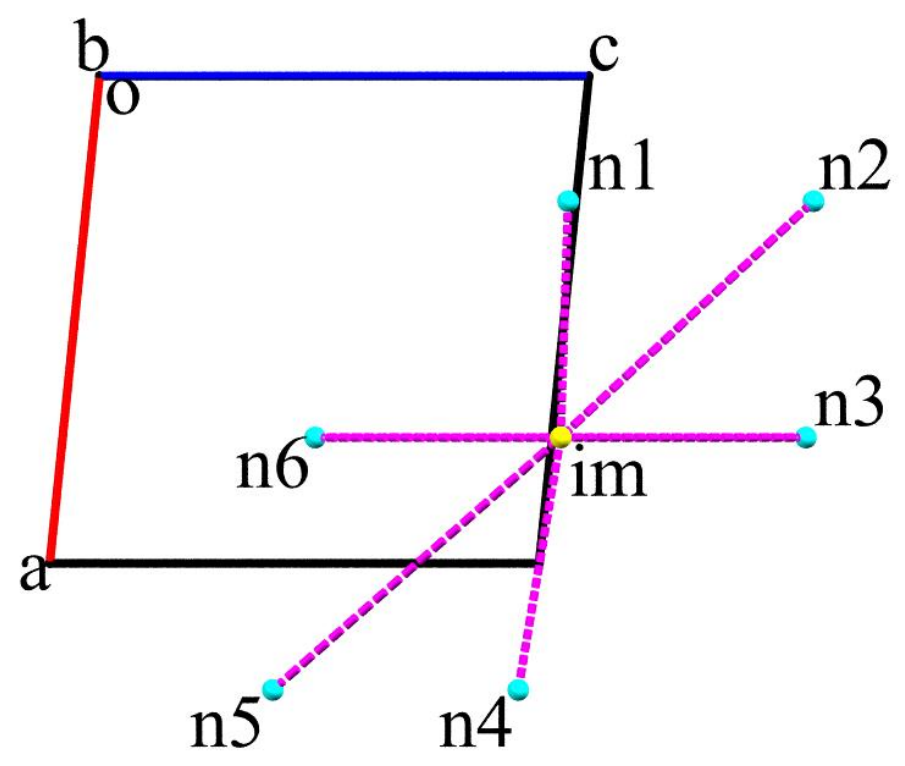

Search for neighbors:

$$
D_{i j} \leq V d W_{i}^{i m}+V d W_{j}^{n m}+1
$$
where $D_{i j}$ - distance between the $i$ and $j$ atoms of the initial molecule and $n$th neighbor $V d W_{i}^{i m}$ - van der Waals radius of the $i$ atom of the initial molecule $V d W_{j}^{n m}$ - van der Waals radius of the $\mathrm{j}$ atom of the neighboring molecule 


\section{Energy-vector diagrams for visualization}

Length of the vector:

$$
L_{n}=R_{n} E_{n} / 2 E_{\text {str }}
$$

where $R_{n}$ - the distance between the geometric centers of $n t h$ neighbor and the initial molecule

$E_{n}$ - interaction energy between between $n t h$ neighbor and the initial molecule

$\mathrm{E}_{\mathrm{str}}$ - energy of the strongest pairwise interaction in crystal

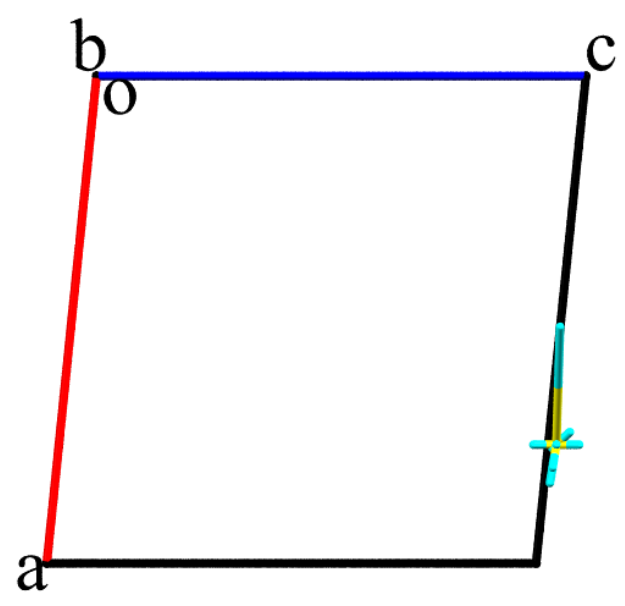

Search for neighbors:

$$
D_{i j} \leq V d W_{i}^{i m}+V d W_{j}^{n m}+1
$$
where $D_{i j}$ - distance between the $i$ and $j$ atoms of the initial molecule and $n t h$ neighbor $V d W_{i}^{i m}$ - van der Waals radius of the $i$ atom of the initial molecule $V d W_{j}^{n m}$ - van der Waals radius of the $\mathrm{j}$ atom of the neighboring molecule 


\section{Energy-vector diagrams for visualization}

Length of the vector:

$$
L_{n}=R_{n} E_{n} / 2 E_{\text {str }}
$$

where $R_{n}$ - the distance between the geometric centers of $n t h$ neighbor and the initial molecule

$E_{n}$ - interaction energy between between $n t h$ neighbor and the initial molecule

$\mathrm{E}_{\mathrm{str}}$ - energy of the strongest pairwise interaction in crystal
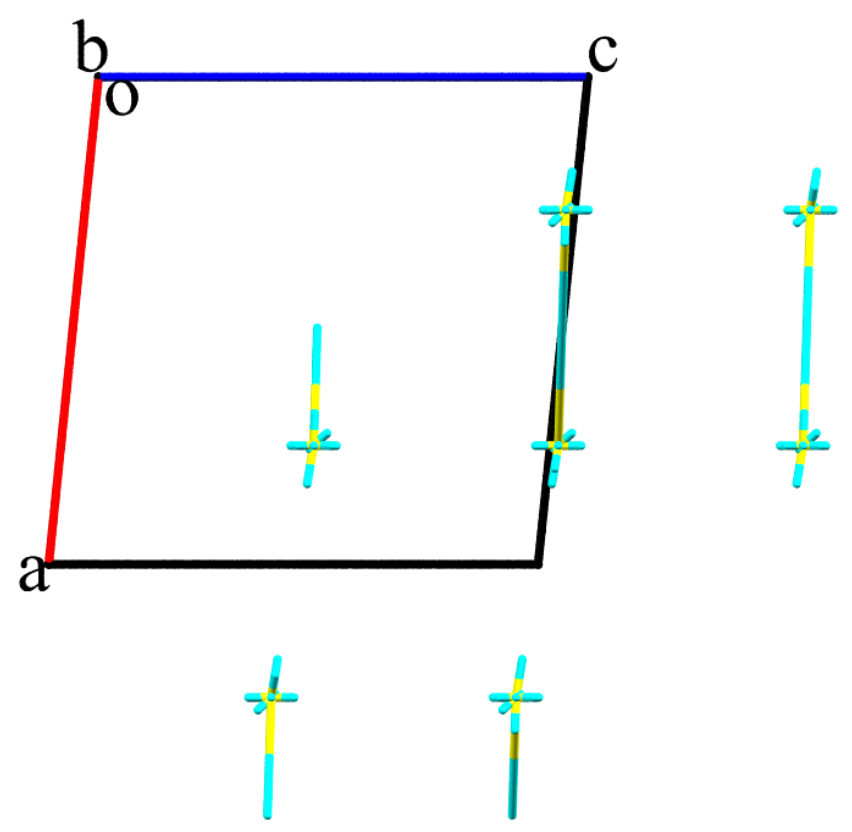
where $\mathrm{D}_{\mathrm{ij}}$ - distance between the $\mathrm{i}$ and $\mathrm{j}$ atoms of the initial molecule and $\mathrm{n}$ th neighbor $V d W_{i}^{i m}$ - van der Waals radius of the $i$ atom of the initial molecule $V d W_{j}^{n m}$ - van der Waals radius of the $\mathrm{j}$ atom of the neighboring molecule

Search for neighbors:

$$
D_{i j} \leq V d W_{i}^{i m}+V d W_{j}^{n m}+1
$$




\section{Energy-vector diagrams for visualization}

Length of the vector:

$$
L_{n}=R_{n} E_{n} / 2 E_{\text {str }}
$$

where $\mathrm{R}_{\mathrm{n}}$ - the distance between the geometric centers of $\mathrm{n}$ th neighbor and the initial molecule

$E_{n}$ - interaction energy between between $n t h$ neighbor and the initial molecule

$\mathrm{E}_{\text {str }}-$ energy of the strongest pairwise interaction in crystal
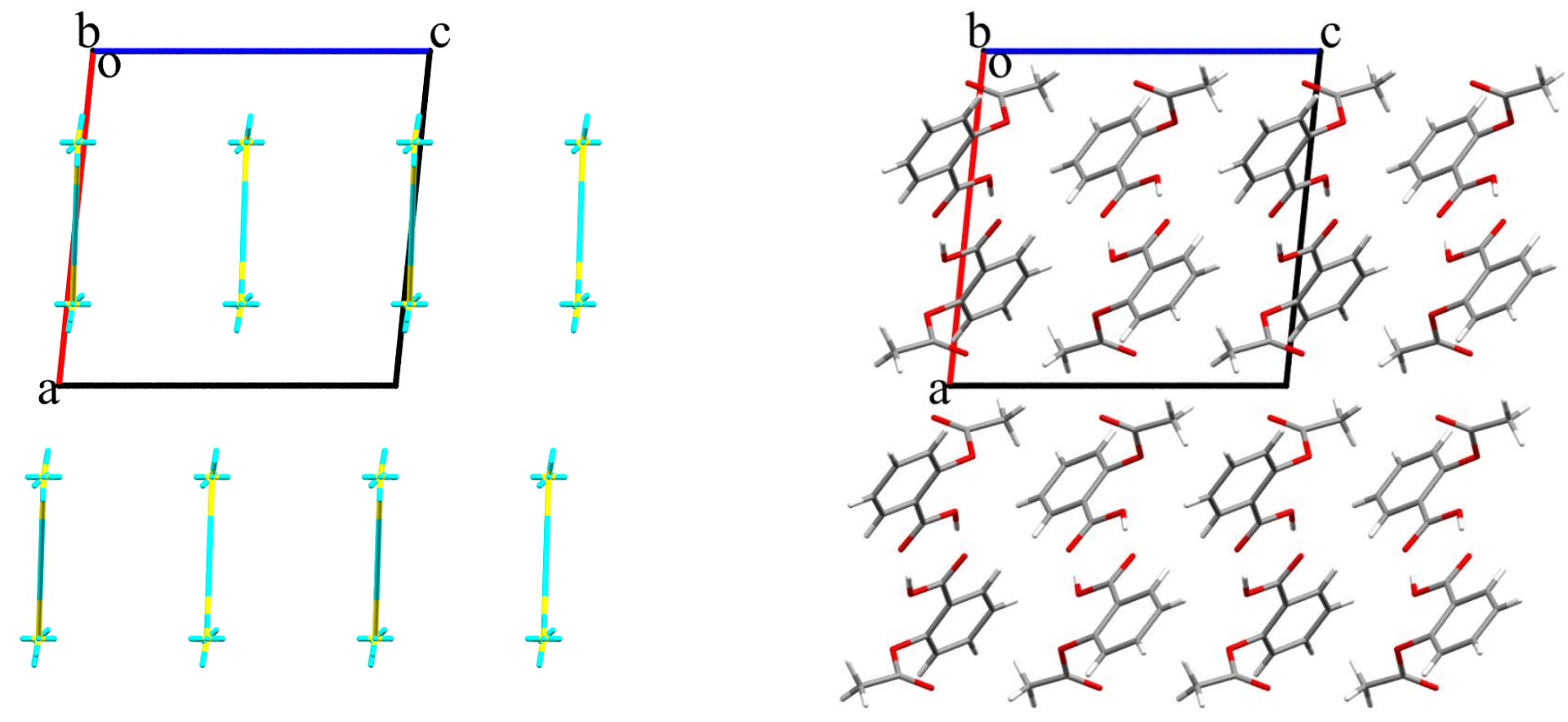

Search for neighbors:

$$
D_{i j} \leq V d W_{i}^{i m}+V d W_{j}^{n m}+1
$$
where $D_{i j}$ - distance between the $i$ and $j$ atoms of the initial molecule and $n t h$ neighbor $V d W_{i}^{i m}$ - van der Waals radius of the $i$ atom of the initial molecule $V d W_{j}^{n m}$ - van der Waals radius of the $\mathrm{j}$ atom of the neighboring molecule 


\section{Classification of molecular crystals}

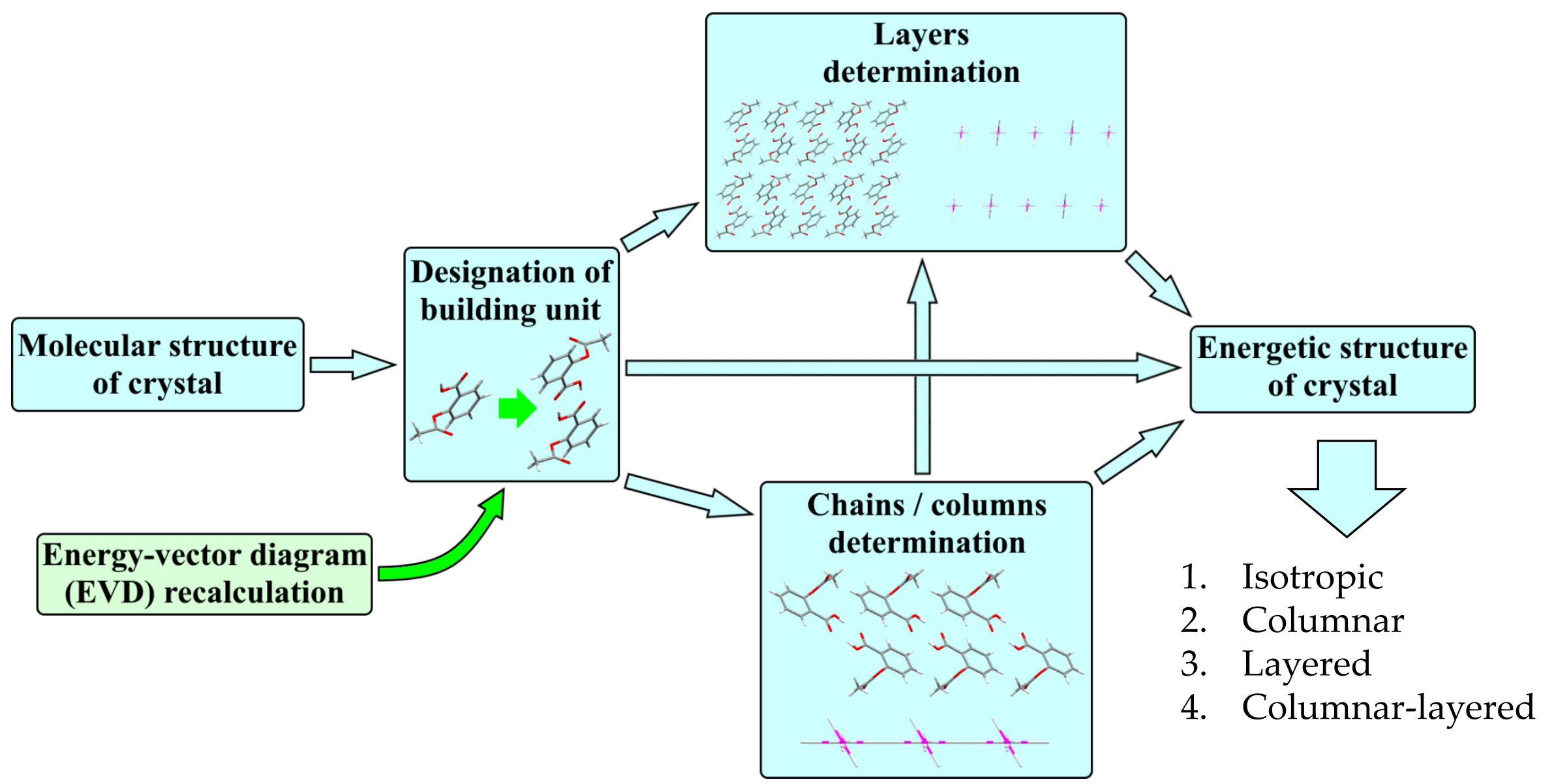

- Relationship between anisotropy of physical properties and anisotropy of crystal packing - Different properties of polymorphs due to different systems of intermolecular interactions 


\section{Aspirin's polymorphic forms}

P21/c: $a=11.2776, b=6.5517, c=11.2741 \beta=95.837$

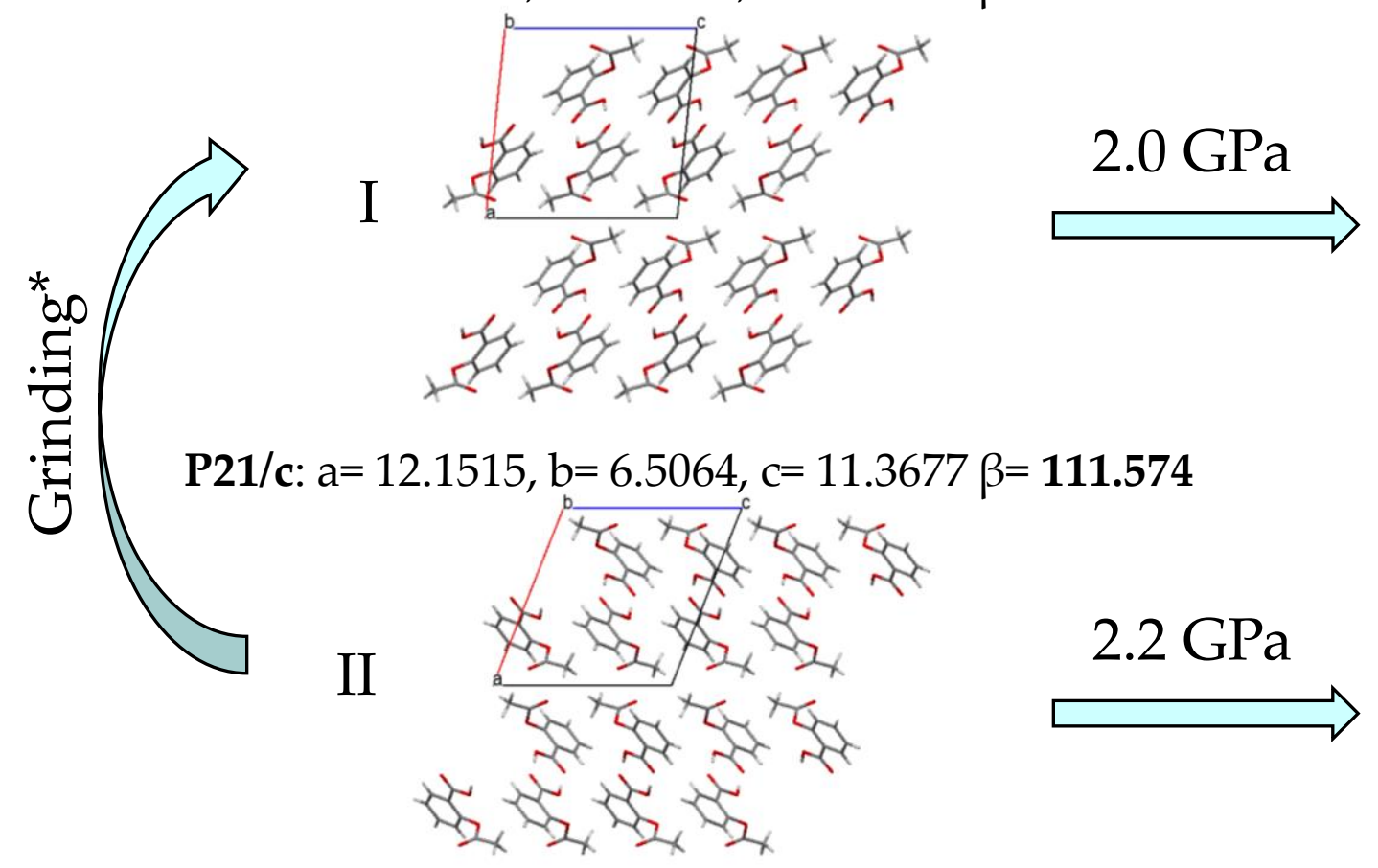

Bond A., Boese R., Desiraju G. (2007).

Angew. Chem. Int. Ed., 46(4), 615-617

P21/c: $a=16.741, b=4.795, c=23.802 \beta=111.08$

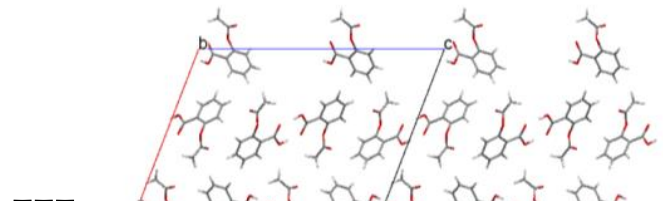

III

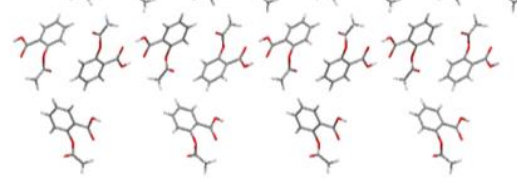

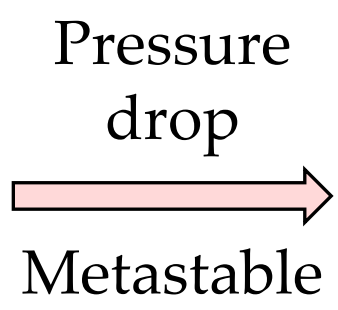

III

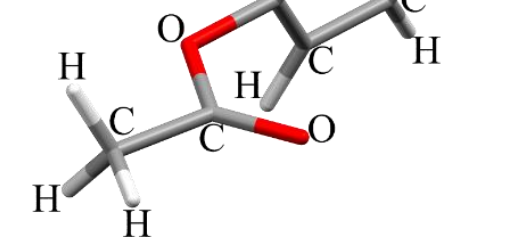

Crystals 2020
*Varughese S., Kiran M. S. R. N., Solanko K. A., [et al.] (2007).

Angew. Chem. Int. Ed., 46(4), 615-617

\section{Unknown form}

Shtukenberg A., Hu C., Zhu Q., [et al.] (2017). Cryst. Growth Des., 17(6), 3562-3566 


\section{Analysis of energetic structure of aspirin's polymorphs}

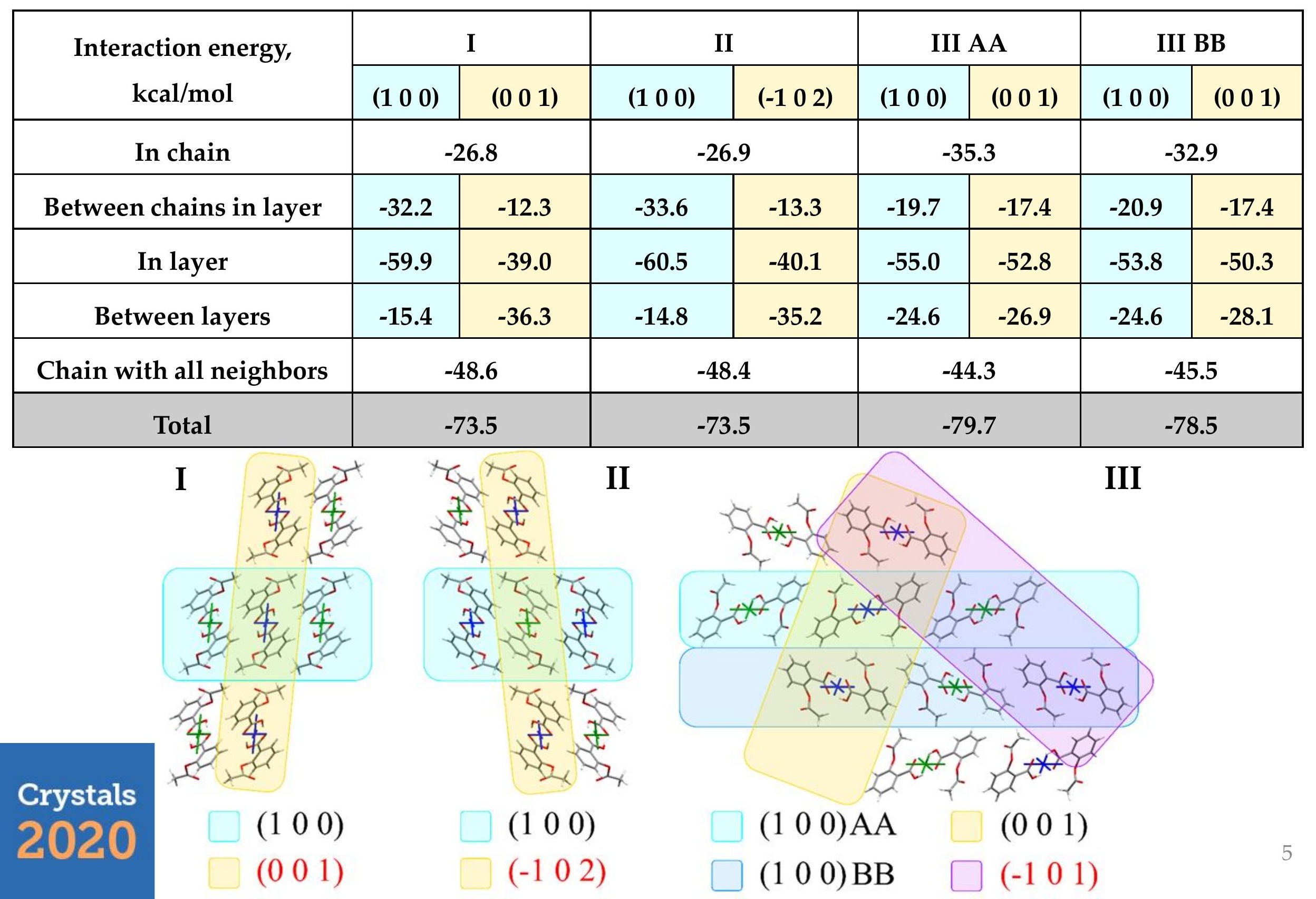




\section{Modelling of shift in aspirin's crystals}

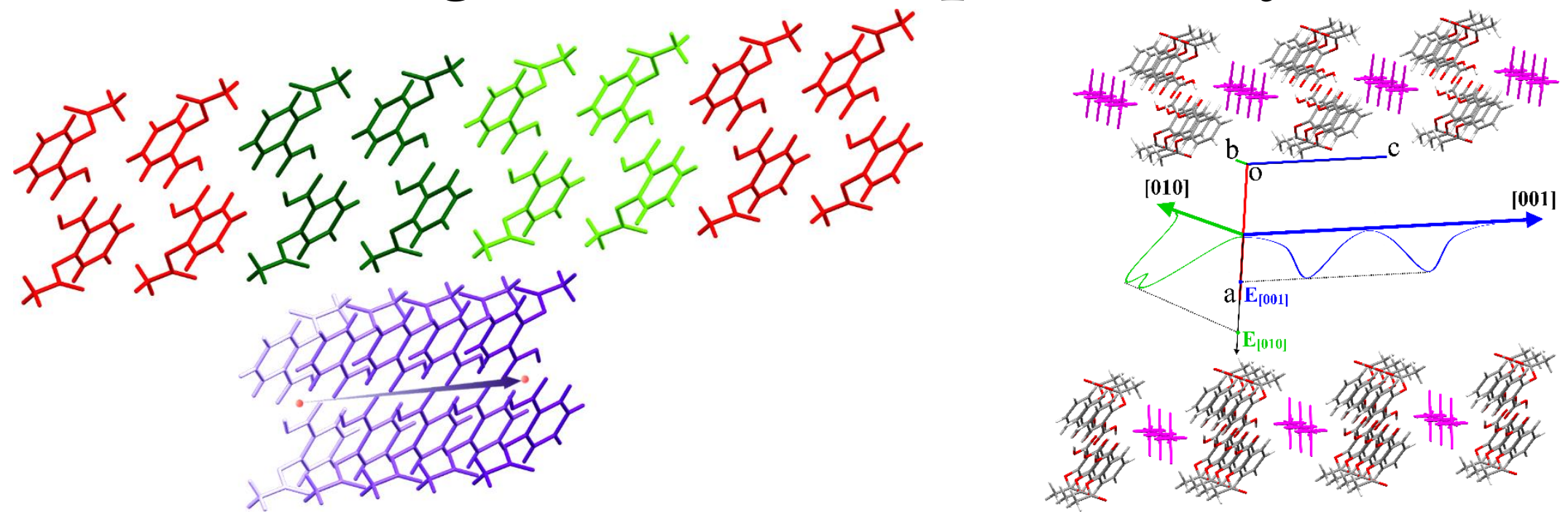

Please, take a look at the video "Shift"

Crystals 2020

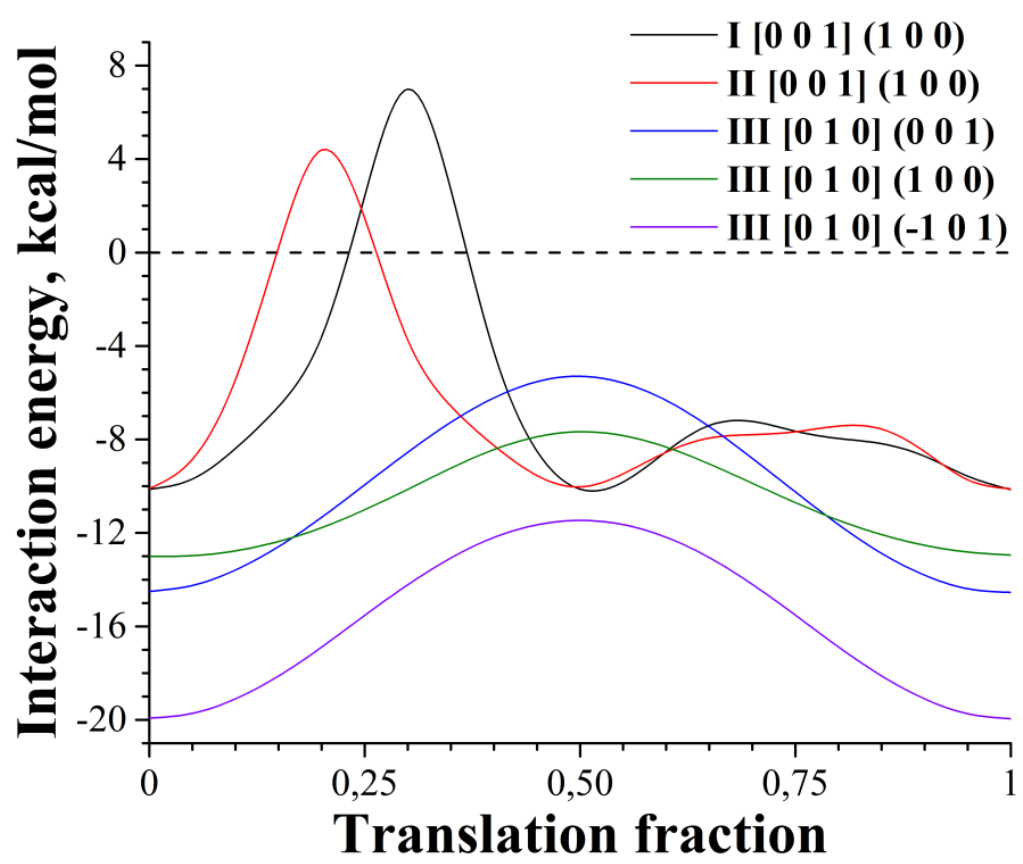

\begin{tabular}{|c|c|c|}
\hline & $\begin{array}{c}\text { Shift energy } \\
\text { barrier, } \\
\text { kcal/mol }\end{array}$ & $\begin{array}{c}\text { Minimal } \\
\text { distance, } \\
\AA\end{array}$ \\
\hline I [001] (100) & 17.1 & 1.19 \\
\hline II [001] (100) & 14.5 & 1.27 \\
\hline III [010] (001) & 9.2 & 1.73 \\
\hline III [010] (100) & 5.3 & 1.73 \\
\hline III [010] (-101) & 8.5 & 1.82 \\
\hline
\end{tabular}

Crystal structure transformation under pressure 


\section{Transformation in piracetam under pressure}
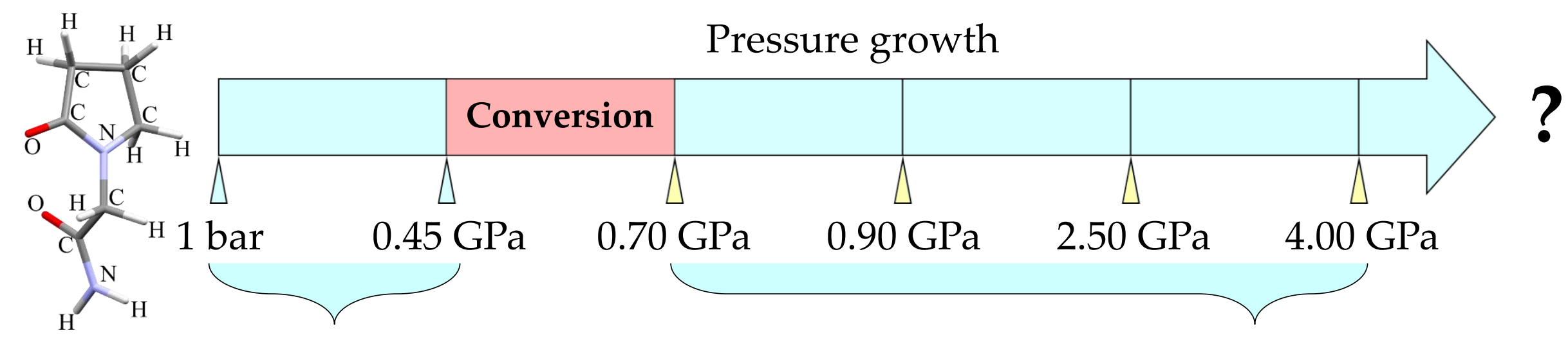



Form II, P-1: $a=6.321, b=6.5597, c=8.380$ $\alpha=79.82, \beta=102.34, \gamma=90.94$
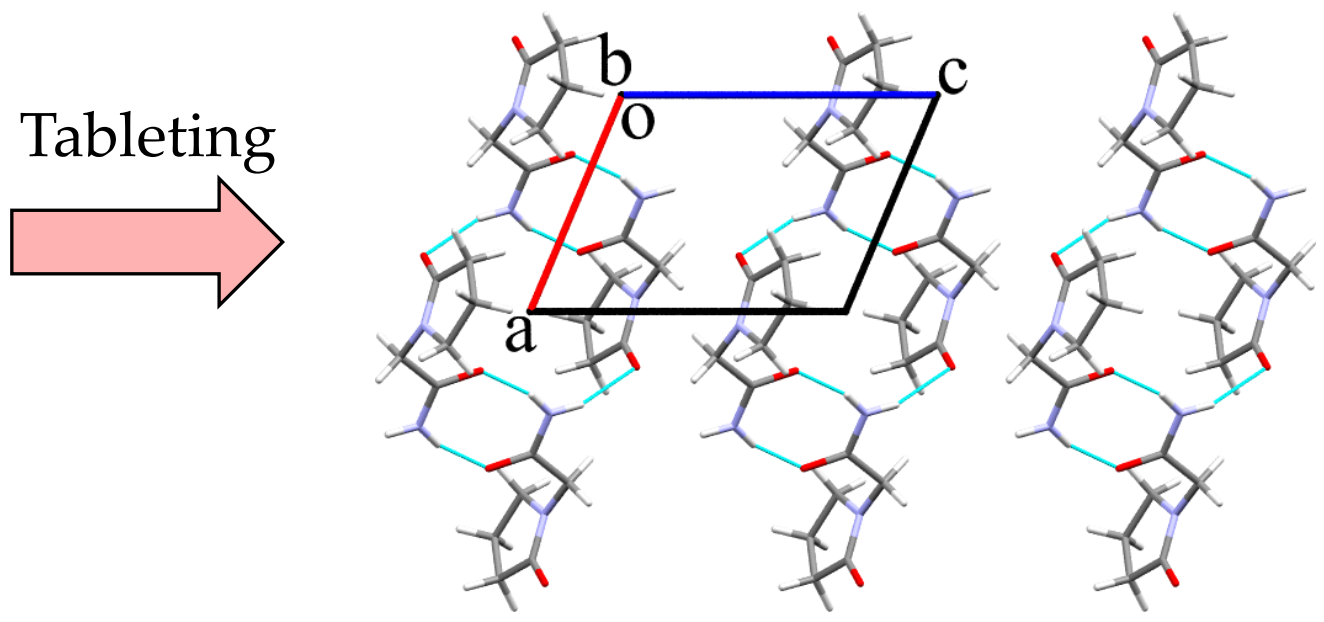

Form V, P-1: $a=6.442, b=6.3530, c=8.737$ $\alpha=81.43, \beta=112.88, \gamma=91.38$ 
Changes in energetic structure of piracetam upon a transition

\begin{tabular}{|c|c|c|c|c|c|c|}
\hline \multirow{2}{*}{$\begin{array}{c}\text { Interaction energy, } \\
\text { kcal/mol }\end{array}$} & \multicolumn{3}{|c|}{ II at 0.45 GPa } & \multicolumn{3}{c|}{ V at 0.70 GPa } \\
\cline { 2 - 7 } & $(001)$ & $(010)$ & $(0-11)$ & $(001)$ & $(010)$ & $(0-11)$ \\
\hline In chain & \multicolumn{3}{|c|}{-35.9} & & -34.6 \\
\hline Between chains in layer & -19.2 & -8.8 & -9.6 & -20.4 & -13.2 & -12.9 \\
\hline In layer & -74.3 & -53.4 & -55.2 & -75.4 & -60.9 & -60.3 \\
\hline Between layers & -18.4 & -28.8 & -27.9 & -26.0 & -33.2 & -33.5 \\
\hline Total & \multicolumn{3}{|c|}{-111.0} & & -127.4 & \\
\hline
\end{tabular}

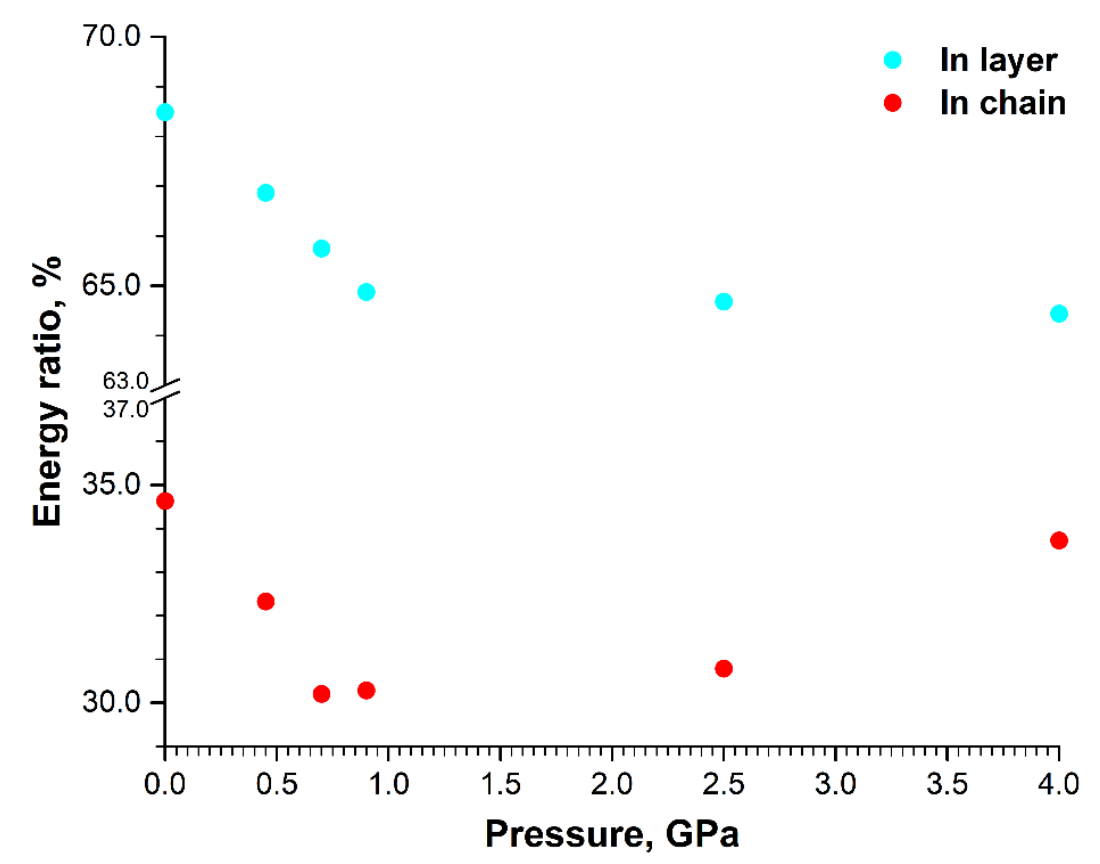




\section{Modelling of shift in piracetam's crystals}
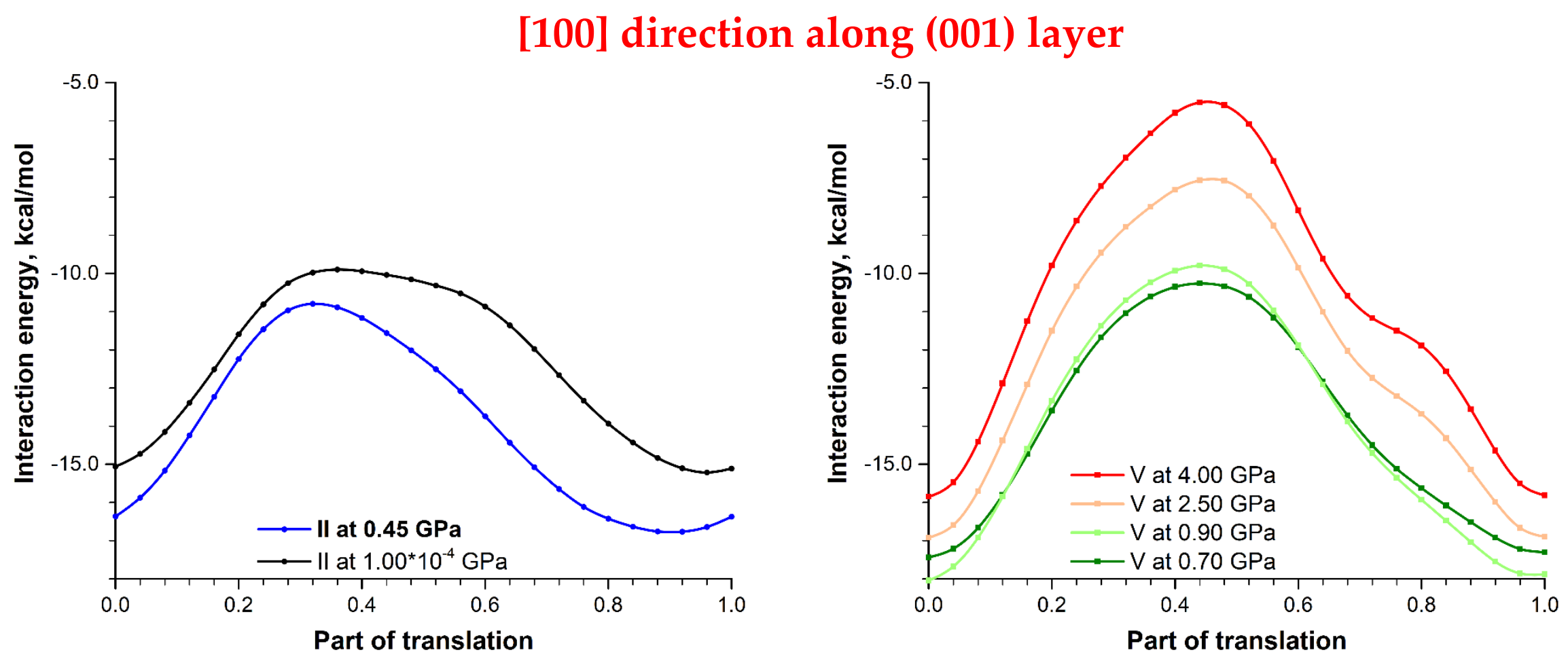

\begin{tabular}{|c|c|c|c|}
\hline Polymorph & $\begin{array}{c}\text { Pressure, } \\
\text { GPa }\end{array}$ & $\begin{array}{c}\text { Shift energy barrier, } \\
\text { kcal/mol }\end{array}$ & $\begin{array}{c}\text { Minimal } \\
\text { distance, } \AA\end{array}$ \\
\hline \multirow{3}{*}{ II } & $\mathbf{1 . 0 0}^{*} \mathbf{1 0}-\mathbf{4}$ & 5.3 & 1.72 \\
\cline { 2 - 4 } & $\mathbf{0 . 4 5}$ & 6.0 & 1.84 \\
\hline \multirow{4}{*}{ V } & $\mathbf{0 . 7 0}$ & 7.2 & 2.01 \\
\cline { 2 - 4 } & $\mathbf{0 . 9 0}$ & 8.2 & 1.96 \\
\cline { 2 - 4 } & $\mathbf{2 . 5 0}$ & 9.4 & 1.88 \\
\cline { 2 - 4 } & $\mathbf{4 . 0 0}$ & 10.3 & 1.84 \\
\hline
\end{tabular}




\section{Cavities detection in piracetam}

Shortening of the van der Waals radii sum at a point:

$$
S v d W R-M D=\min \left(D_{i j}-R_{i}^{F P}-R_{j}^{M P}\right)
$$

where $D_{i j}$ - the distance between the atoms of fixed (i) and motile (j) parts $R_{i}^{F P}, R_{j}^{M P}$ - van der Waals radii of the corresponding atoms in fixed (i) and motile (j) parts

$\begin{array}{lllllllllllllllll}0.0 & 0.1 & 0.2 & 0.3 & 0.4 & 0.5 & 0.6 & 0.7 & 0.8 & 0.9 & 1.0 & 0.0 & 0.2 & 0.4 & 0.6 & 0.8 & 1.0\end{array}$






\section{Cavities detection in piracetam}

Shortening of the van der Waals radii sum at a point:

$$
S v d W R-M D=\min \left(D_{i j}-R_{i}^{F P}-R_{j}^{M P}\right)
$$

where $D_{i j}$ - the distance between the atoms of fixed (i) and motile (j) parts $R_{i}^{F P}, R_{j}^{M P}$ - van der Waals radii of the corresponding atoms in fixed (i) and motile (j) parts

$\begin{array}{lllllllllllllllll}0.0 & 0.1 & 0.2 & 0.3 & 0.4 & 0.5 & 0.6 & 0.7 & 0.8 & 0.9 & 1.0 & 0.0 & 0.2 & 0.4 & 0.6 & 0.8 & 1.0\end{array}$

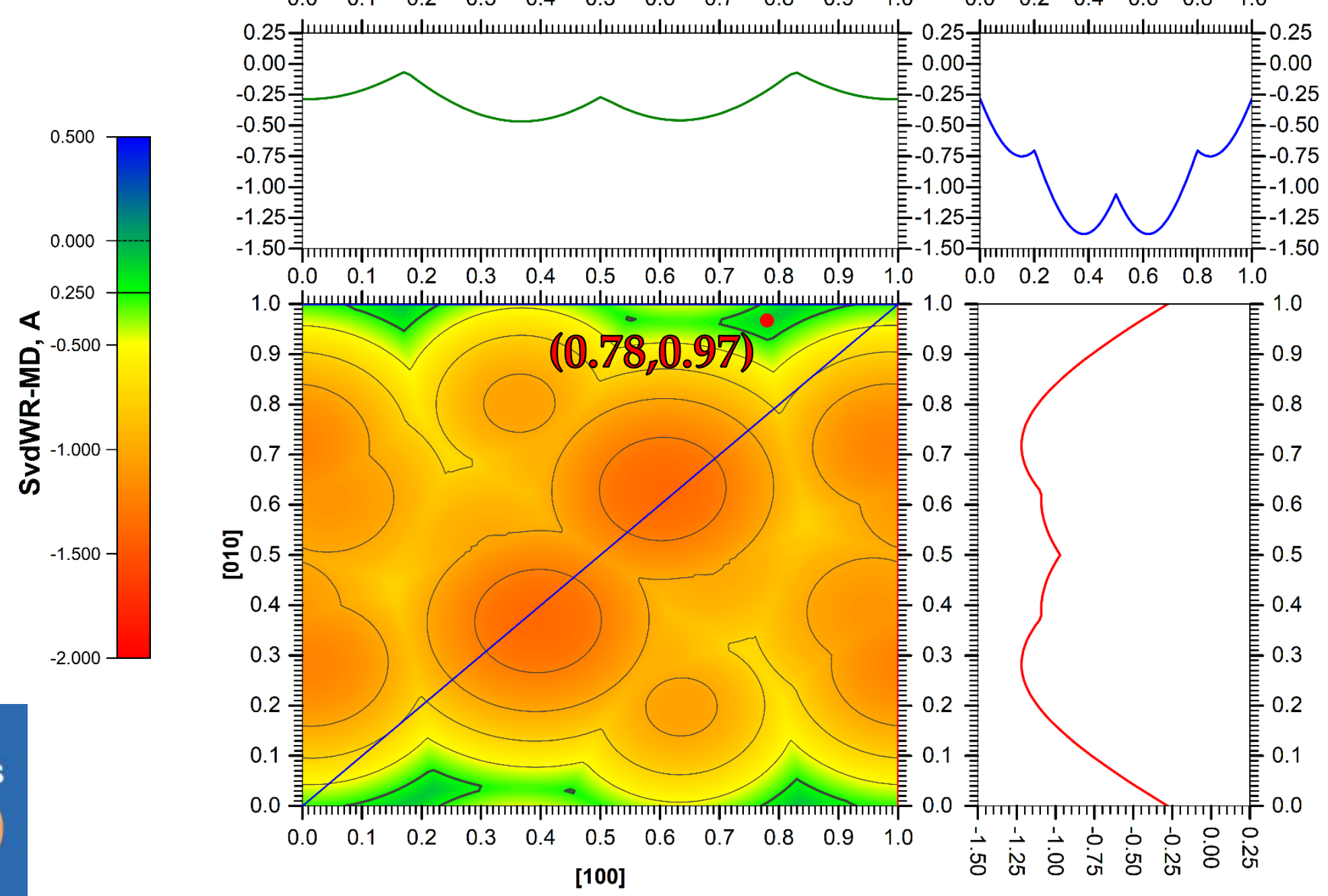


Comparison of structure and energy assessment of the transition in piracetam crystals

[100]

\section{[100]}

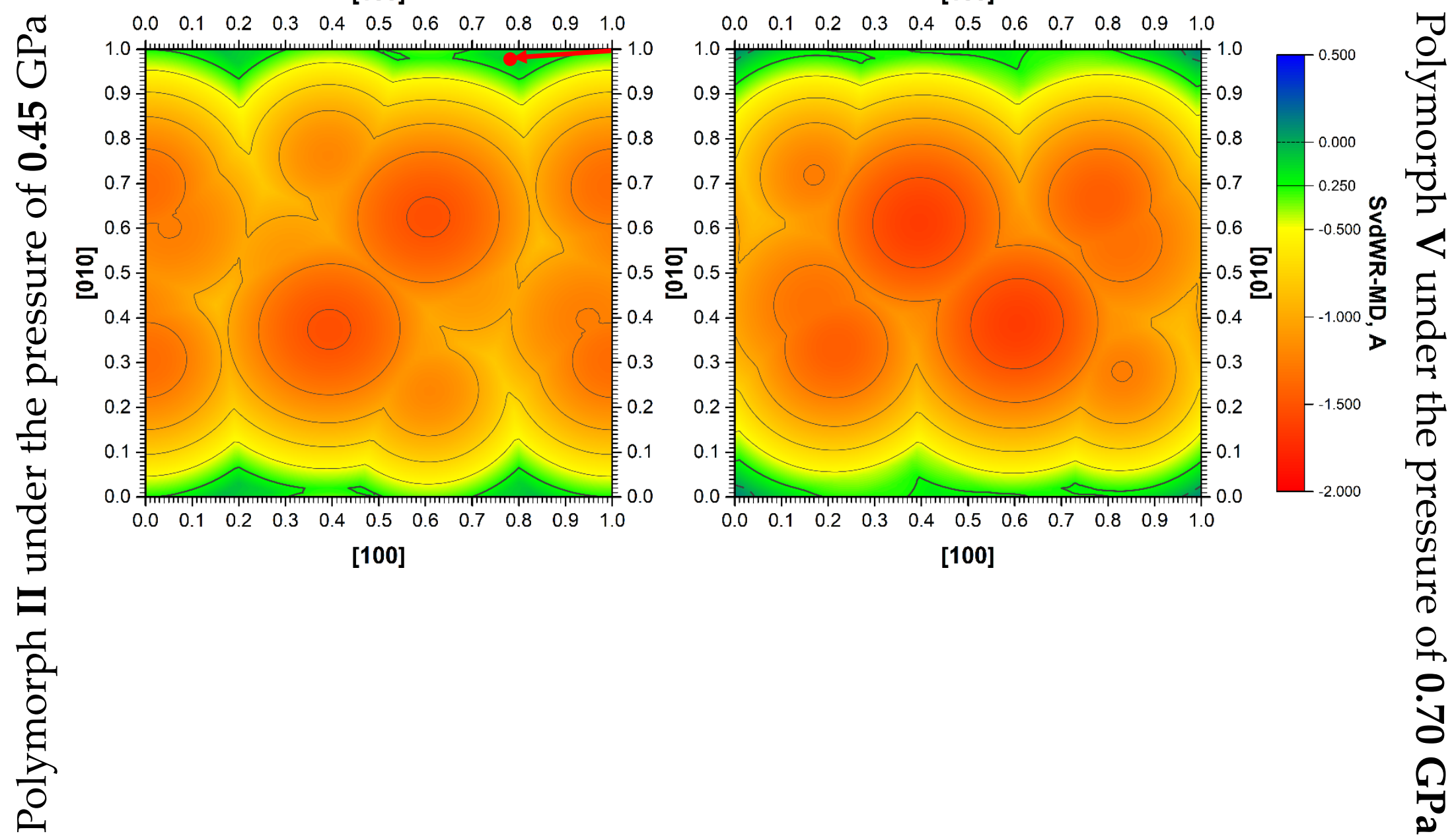

Crystals 
Comparison of structure and energy assessment of the transition in piracetam crystals

[100]

\section{[100]}

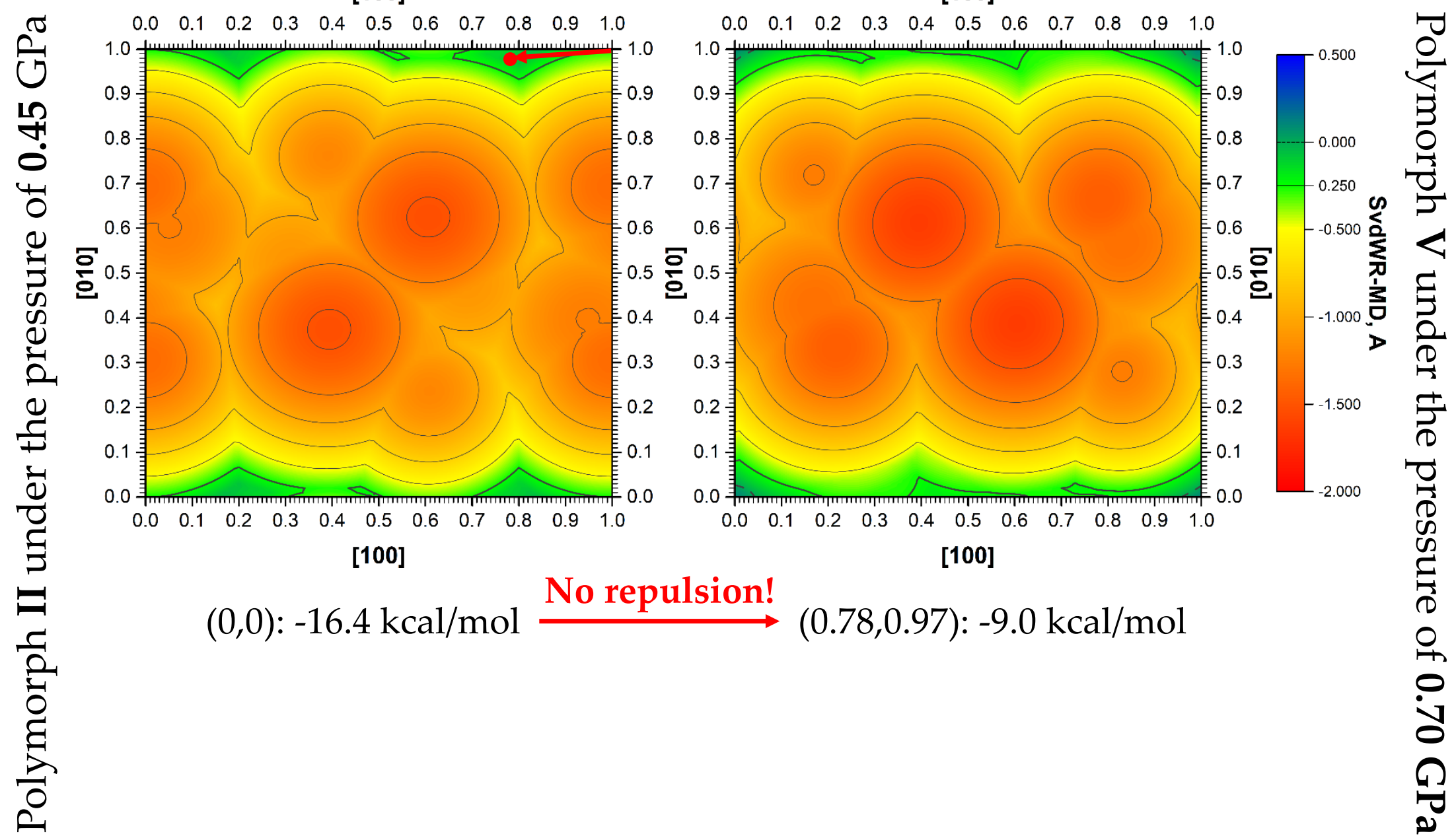

Crystals 
Comparison of structure and energy assessment of the transition

[100] in piracetam crystals






\section{Consistency of ibuprofen's structure}
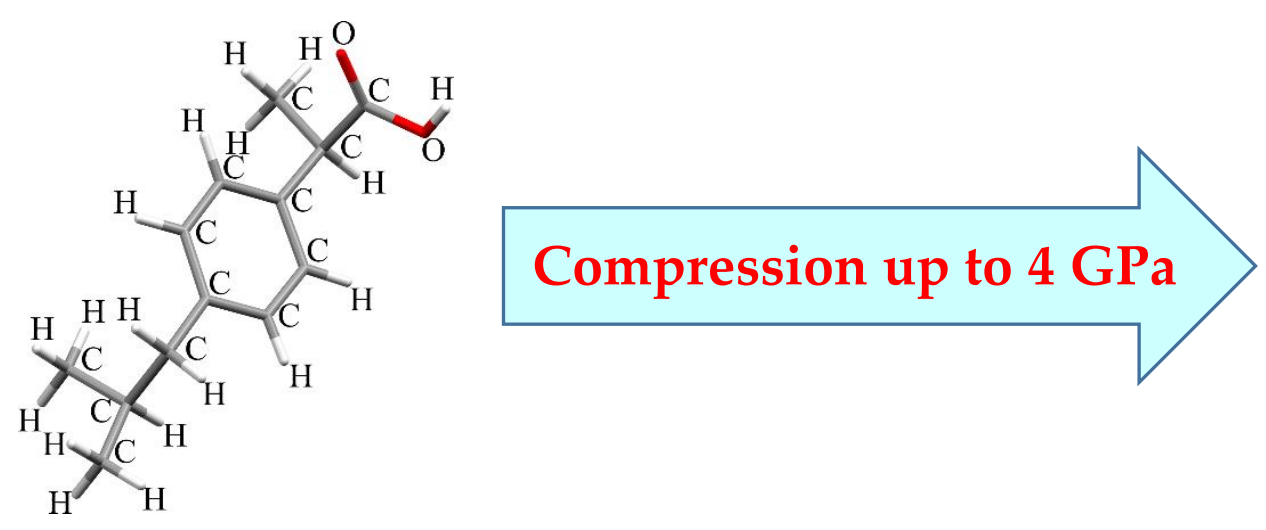

No conversion under pressure!
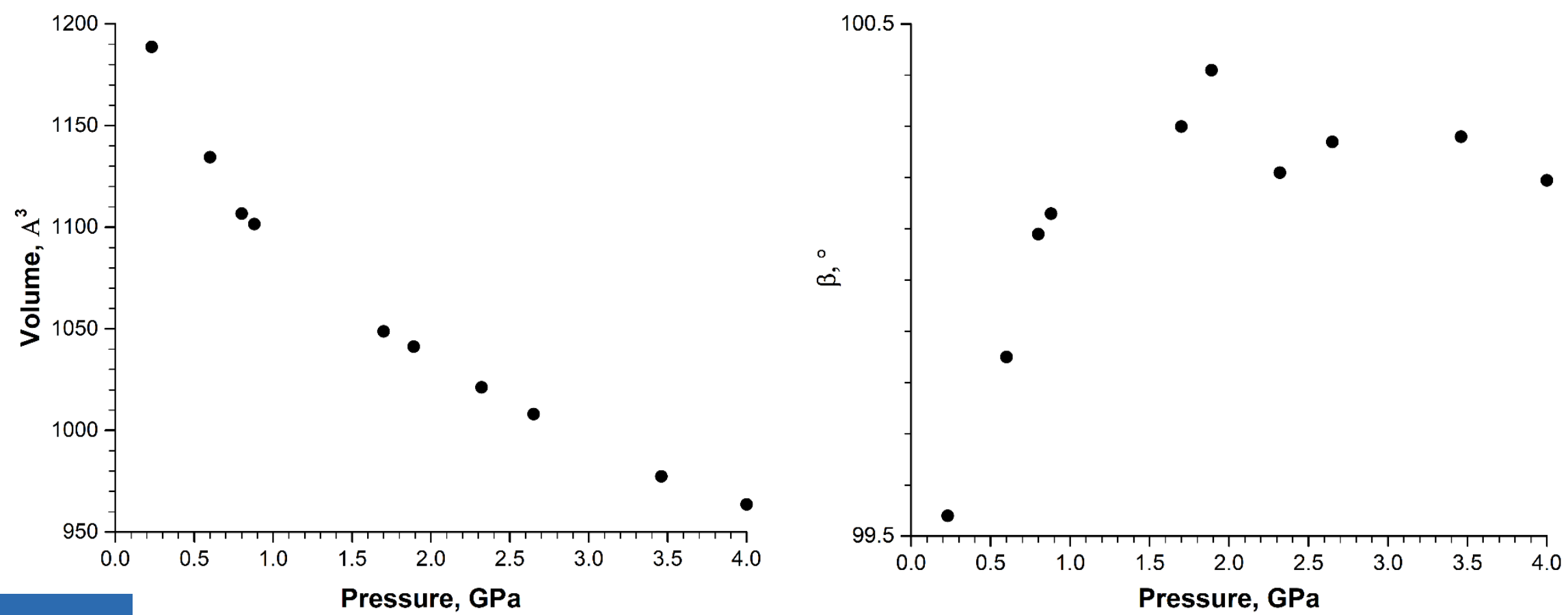

Crystals Ostrowska K., Kropidłowska M., Katrusiak A. (2015) 


\section{Sustainability of ibuprofen's energetic structure}

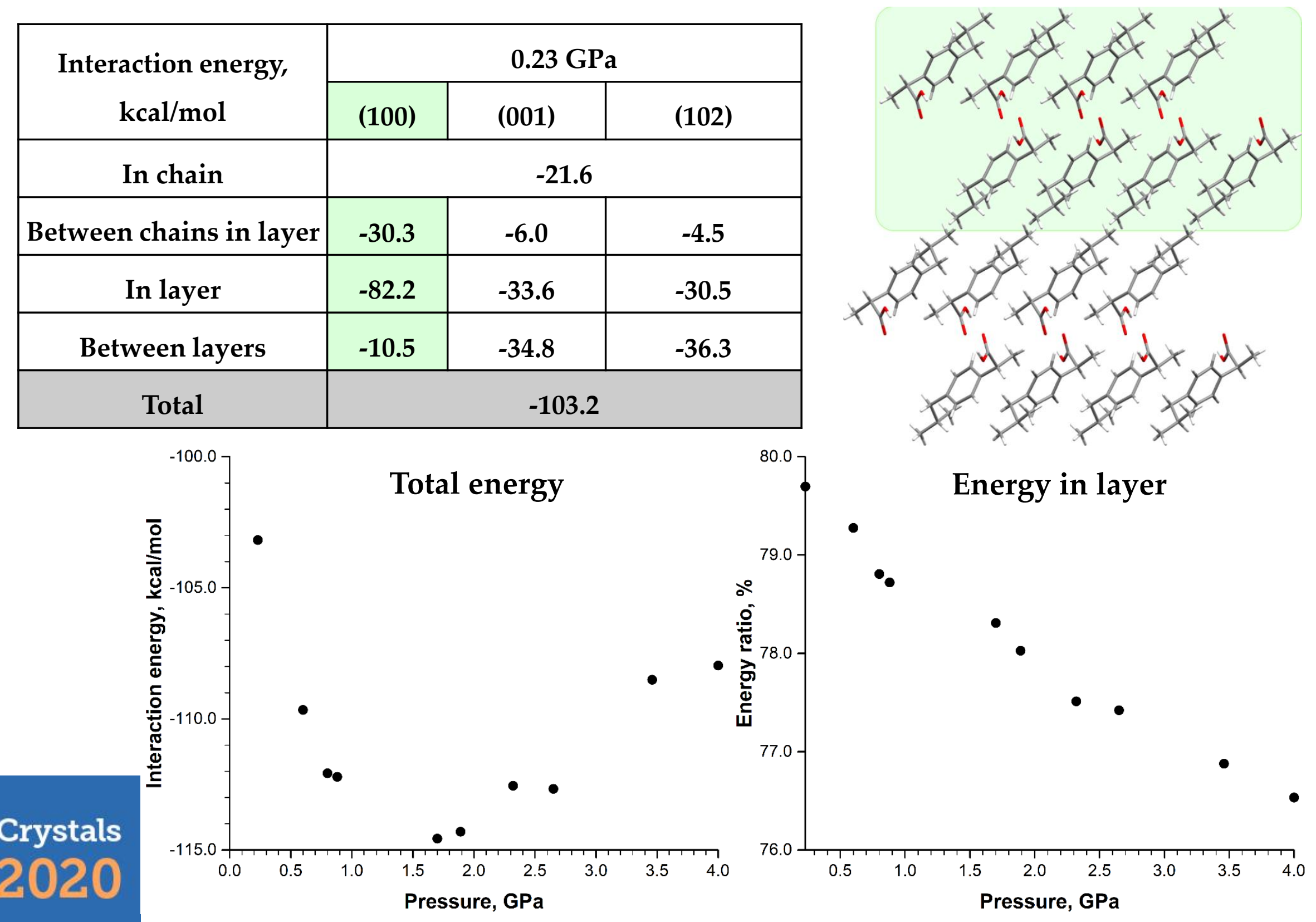




\section{Modelling of shift in ibuprofen's crystals}

[010]
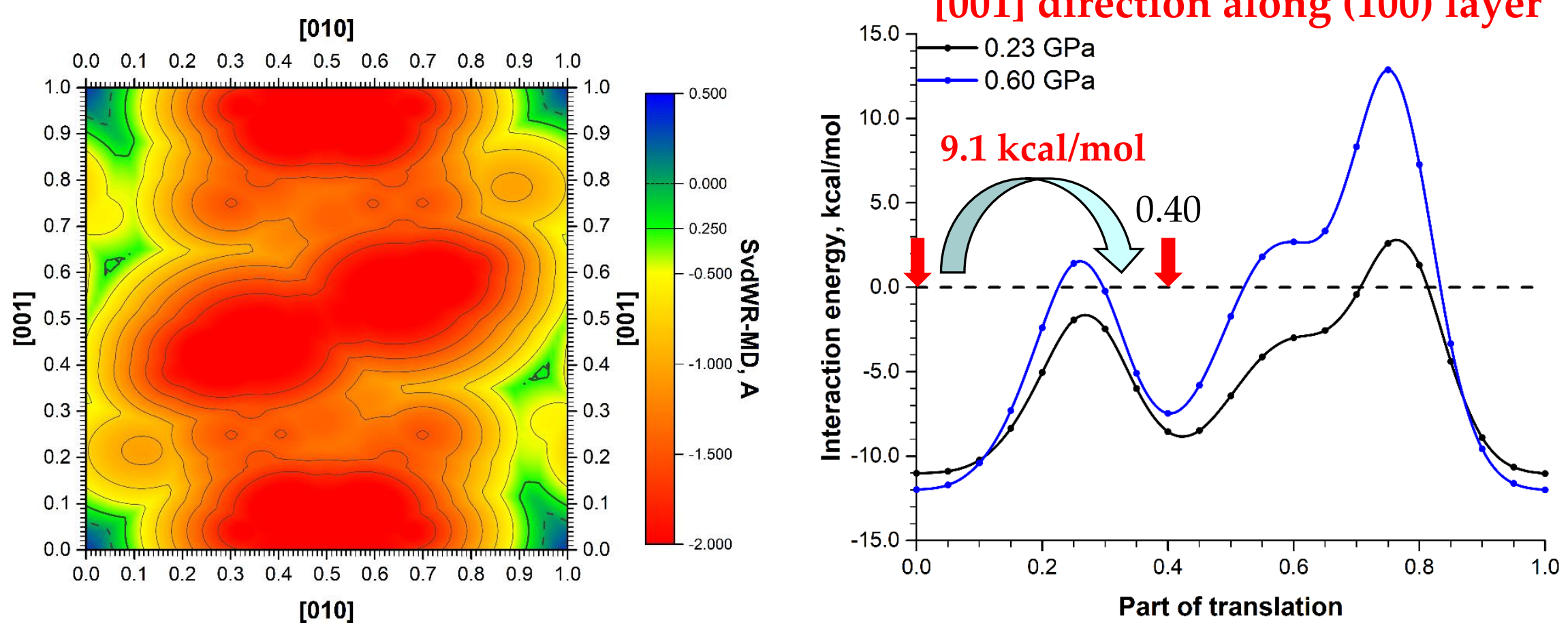

Crystals 2020

\begin{tabular}{|c|c|c|}
\hline Pressure, GPa & Shift energy barrier, kcal/mol & Minimal distance, $\AA$ \\
\hline 0,23 & 13,6 & 1,46 \\
\hline 0,60 & 24,9 & 1,30 \\
\hline 0,80 & 34,8 & 1,18 \\
\hline 0,88 & 34,7 & 1,20 \\
\hline 2,65 & 104,6 & 0,86 \\
\hline 4,00 & 190,7 & 0,70 \\
\hline
\end{tabular}




\section{Conclusions}

* On the base of a study of pairwise interactions energies in crystals the new quantum-chemical method able to describe shear-based transformations of crystal structures was proposed.

* The new cheap express method for the 2 d-detection of cavities in structure was offered and applied for the preliminary assessment of the molecular slips probability in crystals.

* Testing with experimental results showed a good match within the simulated and real properties and transformations for three pharmaceutic compounds: aspirin, piracetam and ibuprofen.
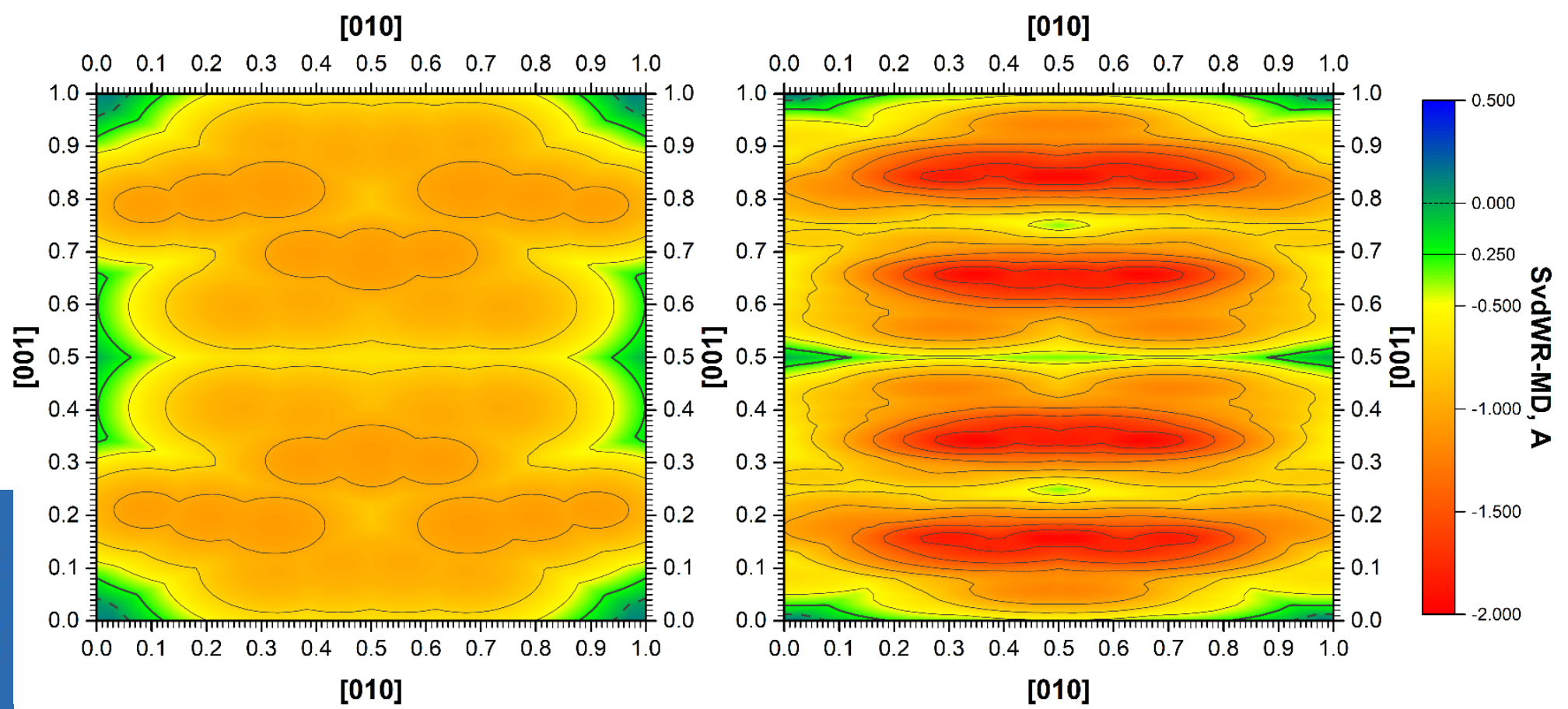